\title{
Participación política de las mujeres en Ecuador ¿Se reduce la brecha?
}

\section{Women's political participation in Ecuador Is the gap reduced?}

\author{
Juan Francisco Trujillo Guerrero
}

Recepción: 10 de septiembre de 2018

Aceptación: 02 de octubre de 2018

\begin{abstract}
Resumen
El objetivo de este trabajo es evidenciar cómo ha evolucionado la participación de las mujeres en la política del Ecuador en el periodo del 2007 -2017. Para ello se plantea la siguiente pregunta de investigación: ¿Ha incidido la normativa sobre paridad de género en la participación de las mujeres en política durante la última década en Ecuador? En este sentido, se realizará un estudio comparativo que tomará como referencia los datos del poder legislativo, además del ámbito local (municipios) en Ecuador. La metodología empleada será de carácter exploratorio-descriptivo y permitirá analizar mediante fuentes documentales, si las cuotas de género para cargos de elección popular han redundado en una mayor participación de las mujeres en la vida política del país. Finalmente se aportarán reflexiones sobre la proyección política de cuadros femeninos dentro de las organizaciones políticas nacionales y las posibilidades que tienen para involucrarse en el ejercicio del poder o generar incidencia.

Palabras clave: democracia, mujeres, representación, género, Ecuador.

\section{Abstract}

The objective of this paper is to show how the participation of women in the politics of Ecuador has evolved during the last years. To this end, the following research question is posed: Has the regulation on gender parity influenced the participation of women in politics during the last decade in Ecuador? A comparative study will be carried out, taking as a reference the data of the legislative branch as well as the local administration (municipalities) in Ecuador. The methodology has an exploratorydescriptive nature and allows to analyze if the gender quotas for positions of popular election have resulted in a greater participation of women in the political life of the country. Finally, reflections will be provided on the political projection of female cadres within national political organizations and the possibilities they have to get involved in the exercise of power and the generation of advocacy.
\end{abstract}

Key words:_democracy, women, representation, gender, Ecuador. 


\section{Introducción}

La participación de las mujeres en la política ecuatoriana, ha tenido una evolución en ciertos espacios durante los últimos años. El país cuenta con una ley de cuotas que regula la paridad y equidad de representación política para cargos de elección popular desde 1997. Si tomamos como referencia los datos del poder legislativo, además del ámbito local, es posible obtener datos importantes para analizar si la normativa ha redundado en una mayor participación de las mujeres en la vida política del país.

La situación de las mujeres en el Ecuador se ha analizado a través de estudios con diferentes aristas durante los últimos años. Los estudios incluyen perspectivas confluyentes como Derechos Humanos, participación política, (Consejo Nacional Electoral, 2014) equidad laboral y género (ONU Mujeres, 2018). De igual forma se creó una agenda Nacional de Mujeres para el período 2013-2017 (SENPLADES, 2014).

El principio de igualdad, "exige considerar, valorar y favorecer en forma equivalente las diferencias en necesidades, intereses, comportamientos $\mathrm{y}$ aspiraciones de mujeres y hombres respecto a los mismos e iguales derechos" (García, 2013: 33).

La igualdad como concepto se entiende desde dos dimensiones fundamentales: la igualdad formal o de jure y la igualdad sustantiva o real. La igualdad formal es la que se encuentra contemplada en las leyes e instrumentos normativos que exponen la igualdad de derechos y oportunidades como el ideal que debe ser cumplido; mientras que, la igualdad sustantiva, es el resultado de la aplicación directa de políticas, planes y programas, que contribuyan al alcance real de oportunidades para todas las personas (SENPLADES, 2014).

\section{Contexto actual en el mundo y América Latina de la participación política de mujeres}

El pasado 13 de marzo marcó el fin de la era de Michelle Bachelet en Chile, quien fue presidenta en dos períodos, el primero entre 2006-2010 y 2014- 
2018, de esta manera se cerró una etapa de referencia: paralelamente, la brasileña Dilma Rousseff (2011-2016) la argentina Cristina Fernández (20072015) y Laura Chinchilla en Costa Rica (2010-2014) ocuparon la cabeza del poder ejecutivo en sus respectivos países marcando un hecho sin precedentes en la región.

Anteriormente, habían sido electas Violeta Barrios en Nicaragua y Mireya Moscoso en Panamá mientras que Lidia Guelier (1979), Rosalía Arteaga (1997) y Ertha Pascal -Trouillot (1990) ejercieron como jefas de Estado interinas en Bolivia, Ecuador y Haití respectivamente. La emergencia de liderazgos femeninos marcó una forma de hacer política en las tres economías más representativas del bloque sudamericano y supuso la expectativa por articular una agenda con demandas surgidas desde los movimientos y organizaciones de defensa de las mujeres.

Un breve repaso del contexto global sobre los indicadores de participación de la mujer en los parlamentos alrededor del mundo alcanza un $23 \%$, el desglose por continentes pone a América Latina a la cabeza con un $28,9 \%$ seguida por Europa con un $27.6 \%$, África Subsahariana con el 23,7\%, Asia 19,8\%, los Estados Árabes 18\%, y el Pacífico 15,6\% (Women in National Parliaments, 2018).

\section{Normativa sobre paridad de género y representación equitativa en el ámbito político}

Uno de los instrumentos internacionales que recogió, expresamente, la necesidad de una participación equitativa de hombres y mujeres en todos los ámbitos de la vida social fue la Convención sobre la Eliminación de todas las formas de Discriminación Contra la Mujer (CEDAW por sus siglas en inglés), suscrita el 3 de septiembre de 1981, tras su ratificación por 20 países. El documento surgido de este encuentro expresa que:

"Los Estados Partes tomarán todas las medidas apropiadas para eliminar la discriminación contra la mujer en la vida política y pública del país y, en particular, garantizando, en igualdad de condiciones con los hombres 
el derecho a votar en todas las elecciones y referéndums públicos y ser elegibles para todos los organismos cuyos miembros sean objeto de elecciones públicas" (CEDAW, 2018).

Otros instrumentos internacionales suscritos por el Estado ecuatoriano son: Plataforma de Acción de Beijing (PAB) (1996); Declaración del Milenio y los Objetivos de Desarrollo del Milenio (2000). Destaca también la Resolución 1325 del Consejo de Seguridad y sus seis resoluciones adicionales sobre mujeres, paz y seguridad 1820 (2008), 1888 (2009), 1889 (2009), 1960 (2010) Security Council Resolution 1960 (2010) 2106 (2013) Security Council Resolution 2106 (2013) y 2122 (2013) Security Council Resolution 2122 (2013). (ONU Mujeres, 2018)

A nivel regional, desde la década de los 90 se impulsaron distintas leyes que apuntaban a la paridad de representación en países como Venezuela, Colombia, Argentina, Ecuador y Perú teniendo como base el artículo 8 de la CEDAW que expresa "Los Estados Partes tomarán todas las medidas apropiadas para garantizar a la mujer, en igualdad de condiciones con el hombre y sin discriminación alguna, la oportunidad de representar a su gobierno en el plano internacional y de participar en la labor de las organizaciones internacionales" (CEDAW, 2018).

\section{El caso de Ecuador}

En 1929 Ecuador se convirtió en el primer país latinoamericano que otorgó el voto facultativo a las mujeres. Desde 1967 es obligatorio para hombres y mujeres. (Espinosa, 2016). Esto marcó una apertura inicial de los espacios formales de participación en política incluyendo el derecho a elegir y ser elegidas

En cuanto a la participación en los procesos electorales, destacan menores niveles de ausentismo en las mujeres. Por ejemplo, para las elecciones seccionales de 2014 el 51,1\% (4'909.340) de las votantes fueron mujeres con un ausentismo del 15,7\% (912.554). Mientras tanto, el 48,9\% (4'691.199) de los votantes fueron hombres con un ausentismo del 19,1\% (1'105.875), de esta forma, puede notarse que el sufragio de las mujeres superó en un 3.4\% 
al de los hombres. En las elecciones generales de 2017 se repitió la tendencia con un 50,1\% de mujeres (5.427.261) y 49,8\% de hombres (5.208.747).

En los últimos tres períodos electorales se han presentado solamente tres candidatas a la Presidencia de la República y 9 candidatas a la Vicepresidencia. En el mismo periodo se registraron 19 candidatos a la presidencia y 15 a la vicepresidencia.

\section{Tabla 1.}

Candidatas y candidatos a la presidencia y vicepresidencia de la República. Período 2009-2017

\begin{tabular}{l|c|c|c}
\multicolumn{1}{c|}{ Elección } & $\mathbf{2 0 0 9}$ & $\mathbf{2 0 1 3}$ & $\mathbf{2 0 1 7}$ \\
\hline $\begin{array}{l}\text { Candidatas y candidatos a } \\
\text { la presidencia }\end{array}$ & 6 hombres & 6 hombres & 7 hombres \\
\cline { 2 - 4 } & 2 mujeres & 0 mujeres & 1 mujer \\
\hline $\begin{array}{l}\text { Candidatas y candidatos a } \\
\text { la vicepresidencia }\end{array}$ & 7 hombres & 2 hombres & 6 hombres \\
\cline { 2 - 4 } & 1 mujer & 6 mujeres & 2 mujeres \\
\hline \multirow{2}{*}{ Total } & 13 hombres & 10 hombres & 13 hombres \\
\cline { 2 - 4 } & 3 mujeres & 6 mujeres & 3 mujeres
\end{tabular}

Fuente: Consejo Nacional Electoral (CNE).

La paridad en cuanto a cuotas de género se recogió por primera vez en 1997. En 1996, sin la ley de cuotas, el Congreso Nacional tenía cinco diputadas mujeres de un total de 82 legisladores, lo que significaba el 6,09\%.

La Ley de Amparo Laboral de 1997 estableció un cupo mínimo del 20\% de representación en las listas pluripersonales en las elecciones para diputados nacionales y provinciales que se realizarían el 30 de noviembre de 1998.

La Reforma Constitucional de 1998 contempló la participación equitativa de hombres y mujeres en los procesos electorales en su artículo 102. Además se introdujo la igualdad de oportunidades entre mujeres y hombres, 
el enfoque de género y la participación política de las mujeres (Constitución de la República del Ecuador, 1998).

Por otro lado, la reforma a la Ley de Elecciones o Ley de Participación Política del año 2000 fijó cuotas a partir de un mínimo de 30\% con listas configuradas en forma alternada y secuencial. También determinó un incremento del $5 \%$ adicional en cada proceso electoral hasta llegar a la igualdad en la representación. (Registro oficial, 2018).

Recientemente, la paridad de género se recoge en la Ley Orgánica Electoral y de Organizaciones Políticas del Ecuador, vigente desde 2009, que en su artículo 160 expresa:

"Las y los representantes de la Asamblea Nacional, representantes ante el Parlamento Andino, consejeras y consejeros regionales, las y los concejales distritales y municipales y vocales de juntas parroquiales rurales se elegirán votando por las candidaturas de las listas establecidas mediante procesos democráticos internos o elecciones primarias y que deberán mantener de forma estricta la equidad, paridad, alternabilidad y secuencialidad entre hombres y mujeres o viceversa" (Código de la Democracia, 2012).

\section{Datos en el poder legislativo}

En las elecciones de 2007 el Parlamento estuvo constituido por un 25\% de mujeres, el número creció en 2009 hasta el 32\% y en 2013 al 38\%, impulsado por el aumento progresivo previsto por la ley de cuotas que era de un $5 \%$ en cada nuevo proceso electoral.

Los resultados oficiales del Consejo Nacional Electoral (CNE) determinaron que entre los 137 legisladores de la nueva Asamblea para el periodo 2017-2021, 52 sean mujeres (Unión Interparlamentaria, 2018).

Alianza PAIS (AP), antes de la ruptura interna que provocó la división del movimiento en dos facciones, tenía el mayor número de parlamentarias: 35. Lo seguía de lejos la ex alianza CREO-SUMA, con siete mujeres. El 
Partido Social Cristiano-Madera de Guerrero (PSC-MG) con seis legisladoras y el Bloque de Integración Nacional (BIN) con tres. En el caso de las 13 comisiones la situación de paridad es así: hay cinco presidentas y seis vicepresidentas. En dos comisiones, la de Relaciones Internacionales y la de Fiscalización, hay dos presidentas y vicepresidentas. Mientras en la Comisión de Desarrollo Económico y la de Régimen Económico, consideradas las más importantes, no hay ninguna mujer de titular ni segunda a bordo.

Para aquellas elecciones, según datos del Consejo Nacional Electoral, se inscribieron 3,793 candidaturas entre principales y suplentes, de los cuales 16 fueron para los binomios presidenciales, 165 para parlamentarios andinos, 450 para asambleístas nacionales, 132 para asambleístas del exterior y 3,030 para asambleístas provinciales. De los 3,793 inscritos, 1,769 (46,66\%) fueron candidatas mujeres y los 2,023 restantes fueron hombres $(53,64 \%)$.

Las mujeres que más se inscribieron para participar fueron jóvenes. Las estadísticas del CNE indican que hay dos legisladoras menores a los 29 años. El 53\% de mujeres oscilan entre los 30 y 39 años; el porcentaje de hombres en ese rango de edad es del $46 \%$. En esta nueva Asamblea hay más hombres que están entre 60 años y más, que mujeres.

De 24 provincias, en ocho no hay representación de la mujer como legisladoras principales: Bolívar, Carchi, Chimborazo, Morona Santiago, Orellana, Pastaza, Sucumbíos y Zamora Chinchipe. Al igual que en las circunscripciones de América Latina, el Caribe y África. (CNE, 2018)

Las estadísticas demuestran que existe una mayor presencia de las mujeres en el legislativo, Sin embargo, hasta agosto de 2016, de 96 proyectos presentados por las asambleístas, solo dos pasaron a trámite y fueron aprobados. A pesar de que el poder legislativo contó con el liderazgo de 3 mujeres no fue posible articular una agenda concreta que priorice las demandas de los movimientos de mujeres. Uno de los grandes temas pendientes del periodo legislativo 2013-2017 fue la tramitación de una ley contra la violencia de género, cuyo tratamiento pasó a segundo plano. Uno de los aspectos positivos fue la conformación de un grupo parlamentario por los derechos de las mujeres. 
Mientras tanto, otras iniciativas como la aprobación del aborto en casos de violación, que había sido impulsado por las máximas autoridades de la Asamblea, provocó la reacción de Rafael Correa, quien amenazó con sanciones disciplinarias en contra de Gabriela Rivadeneira, Rosana Alvarado y Marcela Aguiñaga, coidearias de su partido y proponentes de la iniciativa legal.

En términos porcentuales, la representación de la mujer no ha superado el 38\% en las dos últimas elecciones generales. Tomando en cuenta estos resultados, se puede notar que la paridad de género en la Función Legislativa todavía tiene una brecha de 12 puntos.

Adicionalmente, hay un detalle importante que contribuye a la prevalencia de estas cifras: la mayoría de los movimientos y partidos políticos ubican como cabeza de lista a la Asamblea Nacional a hombres. Si tomamos en cuenta el hecho de que hay algunas provincias donde el número de dignidades a elegirse es impar, tendremos a menos mujeres participando en el proceso electoral.

\section{Gráfico 1.}

Proporción de escaños ocupados por mujeres en el parlamento nacional (1998-2017)

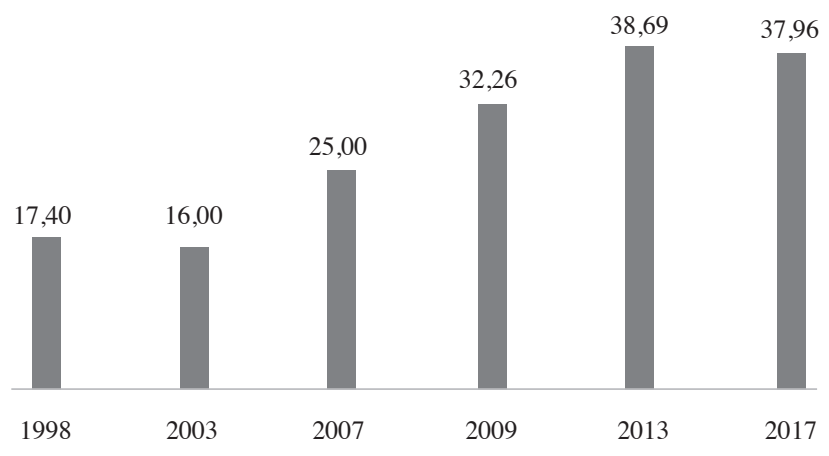

Fuente: CEPAL. 


\section{La brecha en los gobiernos locales}

Más allá de la cantidad de iniciativas legales que se presenten y de que los índices de paridad de género han tenido una evolución positiva en el poder legislativo, la brecha con el nivel local es todavía muy pronunciada, y los indicadores han marchado a un ritmo distinto. Según estadísticas de la CEPAL el porcentaje de mujeres que fueron electas para el cargo de alcaldesa se mantuvo en $2.8 \%$ entre 2000 y 2004 para subir levemente en 2009 ubicándose en 5.9\%. En las últimas elecciones seccionales celebradas en Ecuador en 2014 el porcentaje de mujeres electas fue del 7.2\%.

\section{Gráfico 2.}

Porcentaje de mujeres alcaldesas electas 2000-2014

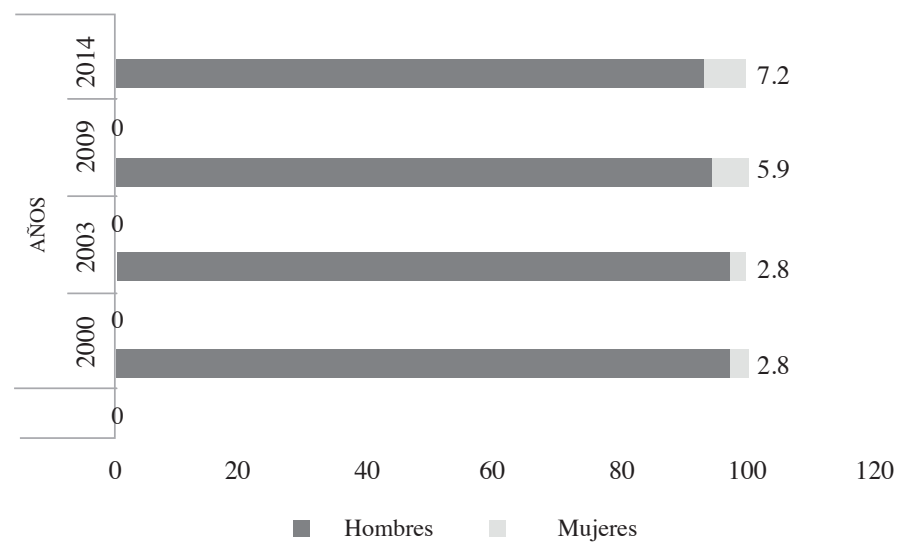

Fuente: CEPAL.

En las elecciones seccionales de 2014, el total de candidatos principales fue de 28.180, de éstos, 16.317 fueron hombres y 11.863 mujeres. Es decir que, en la participación político-electoral, los hombres con un $57,9 \%$, superaron a las 
mujeres con $42.1 \%$ dejando una brecha del $15,8 \%$. El fenómeno es contrario en los porcentajes de candidatos suplentes, en donde la participación de mujeres supera a la de los hombres (Consejo Nacional Electoral, 2014).

\section{Tabla 2.}

Candidatas y candidatos principales según edad y sexo en las elecciones seccionales 2014

\begin{tabular}{|c|c|c|c|c|c|}
\hline \multirow{3}{*}{ DIGNIDAD } & \multicolumn{5}{|c|}{ PRINCIPALES } \\
\hline & \multicolumn{2}{|c|}{ MUJERES } & \multicolumn{2}{|c|}{ HOMBRES } & \multirow{2}{*}{ TOTAL } \\
\hline & CANTIDAD & PORCENTAJE & CANTIDAD & PORCENTAJE & \\
\hline Prefecturas & 16 & $13,80 \%$ & 100 & $86,20 \%$ & 116 \\
\hline Alcaldías & 147 & $12,20 \%$ & 1.054 & $87,80 \%$ & \\
\hline $\begin{array}{l}\text { Concejalías } \\
\text { rurales }\end{array}$ & 1.055 & $42,80 \%$ & 1.410 & $57,20 \%$ & 2.465 \\
\hline $\begin{array}{l}\text { Concejalías } \\
\text { urbanas }\end{array}$ & 2.608 & $45,40 \%$ & 3.137 & $54,60 \%$ & 5.745 \\
\hline $\begin{array}{l}\text { Vocalías } \\
\text { de juntas pa- } \\
\text { rroquiales }\end{array}$ & 8.037 & $43,10 \%$ & 10.616 & $56,90 \%$ & 18.653 \\
\hline TOTAL & 11.863 & $42,10 \%$ & 16.317 & $57,90 \%$ & 28.180 \\
\hline
\end{tabular}

Fuente: Consejo Nacional Electoral (CNE).

Cabe recalcar que las candidaturas para alcaldías no necesariamente guardan relación con la paridad de género, esto debido a su carácter unipersonal. En este sentido la ley electoral de Ecuador solo estipula que ganará la candidata o candidato que obtenga mayor cantidad de votos válidos sin establecer proporcionalidad entre candidaturas de hombres y mujeres. Para alcaldías se presentaron 1.047 candidatos hombres y 147 candidatas mujeres. Como antecedente más reciente, durante las elecciones seccionales de 2014 en Quito, Guayaquil y Cuenca, las más importantes del país, sólo se registró una candidata mujer en cada ciudad.

Algo similar sucede en el caso de prefecto/a viceprefecto/a provincial, pues se garantiza la alternabilidad entre un candidato hombre y una candidata 
mujer o viceversa (art. 163 de la ley electoral ecuatoriana). Según datos del Consejo Nacional Electoral de Ecuador se presentaron un total de 16 candidatas mujeres para el cargo de prefectura provincial mientras que 100 fueron hombres. En las provincias de Pichincha y Guayas todos los candidatos a la prefectura fueron hombres, dejando a las mujeres las plazas para viceprefectura. Solo en Azuay se presentó una candidata para el cargo de prefecta.

Las características de la ley provocan que, por lo general, se deje a las mujeres el rol de viceprefectas, de manera que se cumpla con la ley en su aspecto formal. Actualmente, solo en 2 de las 24 provincias del país son mujeres quienes ocupan las prefecturas. En cuanto a alcaldías son 16 de 221 las mujeres que ocupan esta posición en los concejos municipales.

Estos datos nos permiten establecer que en elecciones locales la participación de mujeres como candidatas a cargos de elección popular no es paritaria y encuentra todavía grandes problemas, pues en cuanto a alcaldías y prefecturas por ejemplo, se mantiene una proporción de 9 candidaturas masculinas frente a 1 femenina. En otros cargos como concejalías urbanas y rurales la proporción es de 6 hombres por cada 4 mujeres. (Consejo Nacional Electoral, 2014).

\section{Tabla 3.}

Candidatas principales en las elecciones seccionales de 2009 y2014

\begin{tabular}{l|r|c|r|c}
\multirow{2}{*}{\multicolumn{1}{c|}{ DIGNIDAD }} & \multicolumn{4}{c}{ MUJERES } \\
\cline { 2 - 5 } & $\mathbf{2 0 0 9}$ & Porcentaje & $\mathbf{2 0 1 4}$ & Porcentaje \\
\hline Prefecturas & 17 & $13,20 \%$ & 16 & $13,80 \%$ \\
\hline Alcaldías & 149 & $11,80 \%$ & 147 & $12,20 \%$ \\
\hline Concejalías rurales & 3.592 & $47,10 \%$ & 1.055 & $42,80 \%$ \\
\hline Concejalías urbanas & 1.446 & $44,00 \%$ & 2.608 & $45,40 \%$ \\
\hline Vocalías de juntas parroquiales & 8.374 & $42,70 \%$ & 8.037 & $43,10 \%$ \\
\hline TOTAL & 13.578 & $42,60 \%$ & 11.863 & $42,10 \%$
\end{tabular}

Fuente: Consejo Nacional Electoral (CNE). 
Como se puede apreciar en el comparativo entre 2009 y 2014 en términos absolutos existe una disminución del número de candidatas mujeres en todos los casos, excepto el de concejalías urbanas, donde casi se duplica. Así el total de candidatas bajó de $13.578(42,6 \%)$ a $11.863(42,1 \%)$ entre una elección y otra.

\section{Gráfico 3.}

Porcentaje de Autoridades Principales Electas según dignidad y sexo en las elecciones seccionales 2014

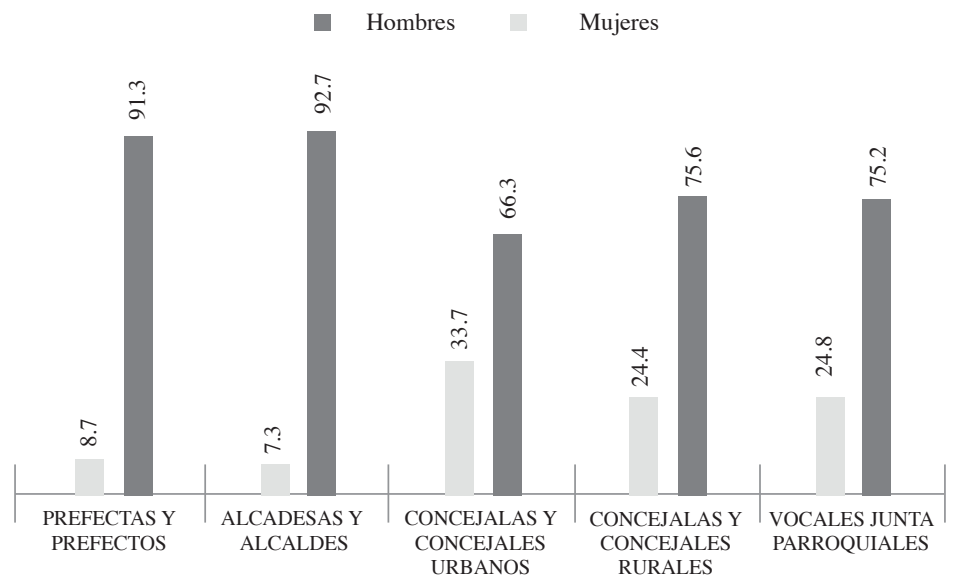

Fuente: Consejo Nacional Electoral (CNE).

Uno de los factores que impide de manera sistemática el incremento de la cantidad de mujeres electas para cargos que salen de listas pluripersonales, es la fórmula de votación mixta, tanto en lista cerrada como abierta, donde la tendencia es elegir más a hombres que a mujeres, aun cuando la lista esté 
conformada de manera alternada y secuencial. De cualquier forma, según la experiencia de otros países con lista cerrada, al votar "en plancha" o lista cerrada, aparentemente se garantiza un porcentaje mayor a favor de las mujeres (CONAMU, 2010).

Evidentemente el espacio en los gobiernos locales es todavía restringido para las mujeres, Otro factor para la prevalencia de este fenómeno es la imposibilidad de sostener procesos a nivel interno en los partidos y organizaciones políticas con miras a garantizar la equidad y paridad en la selección de candidatas y candidatos.

La selección de candidaturas todavía se basa en criterios como la popularidad o el tipo de alianzas que se establecen a nivel local y esto cierra las puertas a la renovación de cuadros políticos, en particular a la representación femenina.

\section{Conclusiones}

De manera general, podemos decir que durante la última década la mayor paridad en cuanto a la representación política de las mujeres no ha redundado en el establecimiento de una agenda nacional que responda a las demandas surgidas desde organizaciones sociales de mujeres.

Como se ha podido observar, las mujeres representan una mayoría de votantes que todavía se encuentra sub representada en los distintos poderes del Estado. Los espacios de interacción política guardan rezagos orientados al machismo y la masculinización. En este sentido las mujeres se ven obligadas a adoptar prácticas de liderazgo "fuerte" para no mostrar debilidad o fragilidad y de esta manera participar en política y obtener el apoyo de partidos y organizaciones en las que los hombres mantienen control.

La conformación de listas, sobre todo en cuanto a la alternabilidad acarrea un problema de representación, pues los vacíos de la ley posibilitan prácticas inequitativas que acentúan la primacía masculina al momento de encabezar listas. Esto se traduce en una mayor cantidad de curules para 
hombres, sobre todo en provincias donde la cantidad de representantes a cargos de elección popular es impar. La inexistencia de procesos de democracia interna también es un factor que limita la paridad de las candidaturas.

La incidencia desde la sociedad civil y organizaciones de base sigue siendo un espacio importante para generar nuevos espacios de incidencia. Es fundamental establecer una hoja de ruta y agenda con temas prioritarios para las mujeres, que deban tratarse desde los distintos poderes del Estado y darles una perspectiva transversal.

Un buen ejemplo de incidencia se dio durante el proceso de socialización del proyecto de Ley Contra la Violencia de Género, la propuesta se desarrolló desde la experiencia de organizaciones como ACDemocracia, organización que trabajó directamente con colectivos de mujeres y recogió sus propuestas en un documento que después fue discutido con un grupo de legisladores y legisladoras quienes avalaron la propuesta para su tramitación definitiva en el seno de la Asamblea Nacional en noviembre de 2017.

Prevalece en el medio un limitado conocimiento sobre igualdad de género y derechos de las mujeres. Muchas mujeres políticas, al igual que sus pares hombres, promueven sus agendas partidistas con una visión neutra o ciega a la igualdad de género. Adicionalmente, se ven forzadas a adoptar actitudes masculinas o machistas.

El ejercicio de diálogo y discusión entre los distintos actores (Estadosociedad-familia) debe llevarse a cabo con objetivos claros y reconociendo que el país avanza siempre en el marco de los instrumentos internacionales de los que es suscriptor.

Las mujeres demuestran liderazgo y capacidad de movilización independientemente de su afiliación política. Es importante cimentar la participación femenina en espacios clave como grupos parlamentarios, que pueden contribuir a la generación de incidencia. Esto permite posicionar en la agenda pública las problemáticas de las mujeres: violencia de género, licencias parentales, equidad laboral, o concientización sobre el trabajo no remunerado. 
También hay otros factores como condiciones de desigualdad estructural, problemas de acceso a la educación, políticas públicas enfocadas en salud sexual y reproductiva o acceso equitativo a nuevas tecnologías que pueden influir en el hecho de que las mujeres participen de forma menos sostenida en la política.

Finalmente, aunque las posibilidades para involucrarse en el ejercicio directo del poder todavía se encuentran limitadas por la confianza del electorado, una mayor articulación del movimiento de mujeres a escala nacional puede asegurar una intervención progresiva en el desarrollo de políticas públicas con enfoque de género, pero para cristalizarlo se deben fortalecer los mecanismos participativos tanto desde las organizaciones políticas como desde los colectivos y organizaciones sociales.

\section{Referencias}

CEPAL. (29 de 07 de 2018). Observatorio de igualdad de género de América Latina y el Caribe. Obtenido de CEPAL: https://oig.cepal.org/es/ indicadores

CONAMU. (2010). Informe sobre el cumplimiento del pacto de Quito. Quito.

Consejo Nacional Electoral. (2012). Código de la Democracia. Quito: El Telégrafo.

Consejo Nacional Electoral. (2014). Indicadores de Participación Política de la Mujer Ecuatoriana. Quito: CNE.

Convención sobre la Eliminación de todas las formas de Discriminación. (18 de Julio de 2018). Convención sobre la Eliminación de todas las formas de Discriminación Contra la Mujer (CEDAW). Obtenido de Sitio Web de la Convención sobre la Eliminación de todas las formas de Discriminación Contra la Mujer (CEDAW): http://www.un.org/ womenwatch/daw/cedaw/text/sconvention.htm 
Espinosa, R. (8 de Noviembre de 2016). "Mujeres en la Política Ecuatoriana". Obtenido de GK: http://elecciones2017.gk.city/2016/11/08/ participacion-politica-de-las-mujeres-en-ecuador/

García, E. (2013). Guía 3 ¿Qué es la política pública con perspectiva de género? Políticas públicas de Igualdad. México: FLACSO .

Ministerio de Relaciones Exteriores y Movilidad Humana. (28 de Julio de 2018). Ministerio de Relaciones Exteriores y Movilidad Humana. Obtenido de Sitio web del Ministerio de Relaciones Exteriores y Movilidad Humana: https://www.cancilleria.gob.ec/wp-content/ uploads/2018/02/politica_para_la_igualdad_de_genero_2018.pdf

ONU Mujeres. (29 de 07 de 2018). ONU Mujeres. Obtenido de Sitio web de ONU Mujeres: http://lac.unwomen.org/es/donde-estamos/ecuador

Registro oficial. (29 de 7 de 2018). OEA. Obtenido de Sitio Web de la OEA: https://www.oas.org/es/sap/deco/moe/ecuador2007/docs/marco\%20 legal/Ecuador-Ley\%20Organica\%20de\%20Elecciones.pdf

SENPLADES. (28 de Julio de 2014). Secretaría Nacional de Planificación y Desarrollo. Quito. Obtenido de Sitio web de SENPLADES: http://www. planificacion.gob.ec/wp-content/uploads/downloads/2014/09/AgendaNacional-de-Mujeres-y-Igualdad-de-Genero.pdf

Unión Interparlamentaria. (8 de Agosto de 2018). Base de Datos sobre Cuotas de Género. Obtenido de Unión Interparlamentaria: https://www.idea.int/ data-tools/data/gender-quotas/country-view/98/35

Women in National Parliaments. (18 de Julio de 2018). Women in National Parliaments. Obtenido de Women in National Parliaments web site: http://archive.ipu.org/wmn-e/world.htm 